\title{
Is there any Relation Between Glucose in Urine with Music Listening During Travel?
}

\author{
Muhammad Imran Qadir and Maria Rizvi* \\ Institute of Molecular Biology and Biotechnology, Bahauddin Zakariya University, Pakistan
}

*Corresponding author: Maria Rizvi, Institute of Molecular Biology and Biotechnology, Bahauddin Zakariya University, Multan, Pakistan.
Received Date: August 11, 2019

Published Date: August 27, 2019

\section{Abstract}

Music can be found almost everywhere being used for entertainment. People also listen to music while traveling. Glucose appears in urine due to hyperglycemia. Basic purpose of this study is finding any relationship between urine glucose and music listening during travel. Urine test was performed for this purpose. $5 \%$ students have glucose in urine and like music in their journey. $77 \%$ students also like music but have no glucose in urine. $18 \%$ students don't like music and have no glucose in urine. Glucose in urine has no relation with music listening during travel.

Keywords: Hyperglycemia; Music listening

\section{Introduction}

Music is used everywhere for entertainment purpose. It can be played at work, home, gym, restaurant, cafes and during travel. People listen to music even for hours. Recent technology of mobiles has made access to music easier than before. As now people can listen to music wherever they are going and these days individuals are spending more time and money on it [1]. Music listening while traveling is a popular activity which is increasing day by day. Approximately $90 \%$ of the transport has musical manifestation. It influences a person traveling in a stress condition. Soft music helps to get rid off from anxiety and feel relaxed. But hard rock or heavy metal music puts negative effect on one's mood [2]. Renal damage of proximal tubules or hyperglycemia causes glucosuria. Normally, all the filtered glucose is reabsorbed by these proximal tubules. Diabetes mellitus and stress causes hyperglycemia. Glucose starts appearing in urine when ultrafiltrate concentration and blood concentration increase from the absorption level of proximal tubules. Detection of glucose is done by enzymatic reaction of glucose oxidase that is specified for glucose. Shelf life of reagent pads is limited. So sunlight protection should be given. If concentration of glucose is low and ascorbic acid is in high concentration in urine than ascorbic acid interferes with this test and false negative results are produced. Formaldehyde also produces false negative results. For false positive results, sample must be contaminated with hypochlorite, chlorine or hypochlorite [3]. Basic purpose of this study is finding any relationship between urine glucose and music listening during travel.

\section{Materials and Methodology}

Required equipment for this test is gloves, urine sample, test strips, watch, clean surface, tissue paper and waste bag. Test was performed on hundred students selected randomly. Gloves were put on before starting the test. Test strip was completely soaked in urine for 2 seconds and then it was extracted from the container having urine sample. To remove the excess urine from the strip it was supported on the container's mouth. Strip was placed on tissue paper to avoid mixing color pads. Time was observed through watch. About after one minute, reactions on reagent strip were appeared. Then this strip was matched with colors of strip container and all the results were recorded. After that all the used materials were thrown in the waste bag and hands were washed completely. Positive results mean there is some abnormality in the person. While negative results mean the person is normal.

\section{Results}

Relation of music listening while traveling with urine glucose is provided in Table 1 . About 2\% male students and 3\% females like music and have glucose in urine while $14 \%$ males and $63 \%$ females also like music but they don't carry glucose in their urine. On the other side, 5\% males and 13\% females don't like music during travel and have no glucose in urine (Table 1). 
Table1: Relation of urine glucose with listening to music while traveling.

\begin{tabular}{|c|c|c|c|c|}
\hline \multirow{2}{*}{ Gender } & \multicolumn{2}{|c|}{ Listen to music while traveling } & \multicolumn{2}{c|}{ Don't listen to music while traveling } \\
\cline { 2 - 4 } & Urine glucose positive & Urine glucose negative & Urine glucose positive & Urine glucose negative \\
\hline Male & $2 \%$ & $14 \%$ & $0 \%$ & $5 \%$ \\
\hline Female & $3 \%$ & $63 \%$ & $13 \%$ & $0 \%$ \\
\hline
\end{tabular}

\section{Conclusion}

Urine glucose has no affinity with music listening while traveling.

\section{Acknowledgement}

None.

\section{Conflict of Interest}

No conflict of interest.

\section{References}

1. Rentfrow PJ (2012) The role of music in everyday life: Current directions in the social psychology of music. Social and personality psychology compass 6(5): 402-416.

2. Dalton BH, Behm DG (2007) Effects of noise and music on human and task performance: A systematic review. Occupational ergonomics 7(3): 143-152.

3. Reine NJ, Langston CE (2005) Urinalysis interpretation: how to squeeze out the maximum information from a small sample. Clinical techniques in small animal practice 20(1): 2-10. 